\title{
ANSPRACHE ZUM ANLASS DER ERÖFFNUNG DER TAGUNG SUCHE NACH DES PUDELS KERN
}

\author{
TOMÁŠ SVOBODA
}

\section{Interkulturelle Kommunikation: Übersetzen und Dolmetschen - Allgemeines zum Studienprogramm}

In diesem Abschnitt möchte ich das Studienprogramm „Mezikulturní komunikace: čeština a němčina pro překlad a tlumočení / Interkulturelle Kommunikation: Übersetzen und Dolmetschen Tschechisch-Deutsch" vorstellen. Es handelt sich um ein Bachelorstudienprogramm, ein Einzelfachstudium, das in Zusammenarbeit des Instituts für Translatologie der Philosophischen Fakultät der Karls-Universität Prag und des Instituts für Slavistik der Universität Leipzig angeboten wird. Das Studium setzt für Studierende im Prager Zweig sehr gute Kenntnisse in Tschechisch und Deutsch voraus, wobei die tschechischen Studierenden bereits über fortgeschrittene Kenntnisse in Deutsch verfügen, als sie in das Programm aufgenommen werden. Nachdem sie sich die Grundlagen der Translatologie, der kontrastiven Linguistik und Literatur- und Kulturgeschichte in den zwei ersten Semestern ihres Studiums angeeignet haben, können sie deshalb bereits das dritte und vierte Semester in Leipzig verbringen. Was die Leipziger Studierenden angeht, ist die Situation recht unterschiedlich. Einige verfügen über gute Vorkenntnisse des Tschechischen, während andere ohne solche Tschechisch-Vorkenntnisse zu studieren beginnen. Deshalb zielt ihre Vorbereitung in dem ersten Semester zusätzlich zu den anderen zu erwerbenden Kompetenzen auch auf die Aneignung der Sprache. Im dritten und vierten Semester vertiefen sie noch ihre Sprach- und Kulturkenntnisse, unter anderem dank der Tandempartnerschaft mit den tschechischen Studierenden, die gerade ihr Mobilitätsjahr an der deutschen Universität verbringen. Erst danach kommen die Leipziger Studierenden nach Prag, also in ihrem fünften und sechsten Semester. Damit verbringen die tschechischen und deutschen Studierenden insgesamt vier Semester zusammen.

Das Studium ist kostenlos, für beide Semester des Auslandsaufenthaltes stehen Stipendien zur Verfügung: Einerseits wird das Studienprogramm von DAAD (Deutscher Akademischer Austauschdienst) unterstützt, andererseits können Stipendienmittel vom Erasmus+ oder dank einer gezielten Unterstützung auch vom Deutsch-Tschechischen Zukunftsfonds in Anspruch genommen werden.

Die AbsolventInnen erwerben während ihres Studiums alle Fähigkeiten und Fertigkeiten auf der Bachelor-Ebene, die sie für eine Ausübung des Übersetzungs- und Dolmetschberufs brauchen. 
Weitere Informationen zum Studiengang können Interviews der beiden Leiter des Studienprogramms (Prof. Dr. Danuta Rytel-Schwarz und PhDr. Bc. Tomáš Svoboda, Ph.D.) entnommen werden: Danuta Rytel-Schwarz im Gespräch für die Philosophische Fakultät der Karls-Universität Prag, $2018^{1}$ und Tomáš Svoboda im Gespräch für dieselbe Fakultät. $^{2}$

\section{Ansprache zum Anlass der Eröffnung der Tagung}

In meiner kurzen Ansprache möchte ich mich zu der Geschichte unseres gemeinsamen Studienprogramms und zu dem, was es so speziell macht, äußern.

Gestatten Sie mir mit einigen Zahlenangaben, eigentlich Meilensteinen aus der bisherigen nahezu 10-jährigen Geschichte unseres gemeinsamen Programmes, zu beginnen.

Die Entstehung unseres gemeinsamen Studienprogramms mit Doppelabschluss (Double Degree) „Mezikulturní komunikace: čeština a němčina pro překlad a tlumočení / Interkulturelle Kommunikation: Übersetzen und Dolmetschen Tschechisch-Deutsch“ (Double Degree, DD) wurde zum ersten Mal 2009/2010 erwogen. Ich habe mir damals Gedanken gemacht, wie wir die Attraktivität unserer germanistischen Studiengänge am Institut für Translatologie steigern könnten. 2012 war es dann so weit mit einem formellen Austausch, als Prof. Danuta Rytel-Schwarz begann, Wege zu suchen, an der Universität Leipzig das damals schon gemeinsame Vorhaben in die Wege zu leiten.

2014 wurde ein gemeinsamer Kooperationsvertrag zwischen der Karls-Universität Prag und der Universität Leipzig unterzeichnet, dessen aktualisierte Version 2019 unterschrieben werden konnte. Die Akkreditierung in Prag fand 2015 statt. Die erste Absolventin aus Leipzig gab es 2018. Im September 2019 hat auch der erste Absolvent aus unserem Prager DD-Zweig sein Studium erfolgreich abgeschlossen. Dies ist eigentlich eine Erfolgsgeschichte, aber auch eine, die mit viel Anstrengung und Entbehren verbunden ist.

Ich möchte jedoch noch in einem weiteren Sinne über Nummern und Ziffern im Zusammenhang mit unserem gemeinsamen Studiengang sprechen. Eigentlich habe ich mich gefragt, was das Typische, das Spezifische sei, was unser gemeinsames Programm ausmacht. Meine Damen und Herren, es ist die Zahlenangabe „Double“, oder „Doppel“: Double Degree, Doppelabschluss, Doppelstudiengang, „studijní program s dvojím diplomem“. Natürlich heißt es in erster Reihe, dass die AbsolventInnen zwei Diplome erhalten, einen an der Prager Karls-Universität, einen an der Universität Leipzig. Aber man kann diese Idee noch weiterentwickeln: „Double“ heißt immer etwas Intensiveres (ein doppelter Espresso), zweimal so Gutes (ein doppelter Genuss, so heißt es oft in den Werbespots für Kulinarisches). Und genau das ist es auch, was unser Programm ausmacht: Die zwei Diplome, das Studium in zwei Sprachen, auf zwei Universitäten, das gewisse Extra... Ja aber auch: Die Studierenden müssen sich (figurativ) zweimal so viel anstrengen, sie verbringen ja zwei Semester im Ausland. Auch Koordinierungsarbeit mit dem Programm gibt es doppelt so viel als gewöhnlich (und viele von uns erinnern sich an die doppelt so

Online unter: https://www.ff.cuni.cz/2018/09/mezi-prahou-lipskem/ [25.11.2020].

2 Online unter: https://www.ff.cuni.cz/2020/01/absolvent-programu-mezikulturni-komunikace-cestina -nemcina-ma-dvojim-diplomem-dvere-otevrene-nemecky-mluvicich-zemich/ [25.11.2020]. 
vielen Schwierigkeiten zu Beginn). Aber es gibt auch eine zweifache Portion positiver Aspekte für die Studierenden und die gemeinsamen tschechisch-deutschen Beziehungen: doppelte Belohnung, sozusagen.

An dieser Stelle möchte ich mich bei allen meinen Kolleginnen und Kollegen aus Prag und aus Leipzig bedanken: Bei Dr. Astrid Winter für ihre Unterstützung, die sie diesem Programm von Anfang an, eigentlich seit den ersten Erwägungen, widmete. Dr. Věra Kloudová und Dr. Petra Mračková-Vavroušová für ihre professionelle Art und Weise, mit der sie sich den Studierenden widmen und sie organisatorisch unterstützen, und natürlich auch bei Dr. Stanislav Rubáš, Institutsleiter der Prager Translatologie, der dem Vorhaben vehement unter die Arme griff, wenn es nötig war. Mein herzliches Dankeschön geht an Dr. Christof Heinz und Mgr. Běla Michálková für ihre Hilfe und tatkräftige Mitarbeit. Und ein „Double“-Dankeschön gebührt Frau Professorin Danuta Rytel-Schwarz für ihr Ausharren und ihre Unermüdlichkeit, mit der sie dieses Programm so aktiv und hilfsbereit an der Leipziger Seite koordiniert und ausgezeichnet betreut.

Zum Schluss möchte ich mich an die Studierenden wenden: Unser Doppelstudiengang ist der erste und bisweilen der einzige seiner Art an der gesamten Philosophischen Fakultät der Karls-Universität, den es auf der Bachelor-Ebene gibt. Im Prager Zweig, wo wir Aufnahmeprüfungen haben, gab es 2019 beinahe 50 BewerberInnen für das Programm und nur etwa $20 \%$ davon konnten aufgenommen werden. Die BewerberInnenzahlen zeigen, dass viele den Mehrwert des Programms wahrnehmen und für Sie, die erfolgreichen KandidatInnen, bedeutet es, diese Chance zu nutzen und Ihr Studium zu einem erfolgreichen Abschluss zu bringen. Auch hier habe ich eine Analogie mit dem doppelten Konzept parat: In einem gewissen (positiven) Sinne führen Sie ein Doppelleben: Sie studieren hier und dort, Sie befassen sich mit zwei Sprachen, zwei Kulturen, Sie werden sagen können, Sie haben einen zweifachen Hintergrund. Ein bekannter Translatologe reiht ÜbersetzerInnen und DolmetscherInnen in dieselbe Reihe mit Diplomaten, denn sie alle sind Grenzgänger, sie alle beteiligen sich an dem gemeinsamen Austausch zwischen zwei Ländern und Kulturen, sie nehmen an der Verständigung Teil. Ich möchte Sie, liebe Studierende, also dazu auffordern, sich dieser Aufgabe als gut ausgebildete Kultur-MediatorInnen bewusst zu sein und ich hoffe, Sie führen sich diese einzigartige Gelegenheit und auch die daraus resultierende Verantwortlichkeit vor Augen, wenn Sie dieses Programm studieren und, eventuell, auch nach dem Abschluss in diesem Gebiet tätig sind.

Und nun: Falls Sie gut zugehört haben, habe ich in meiner Ansprache 10-mal das Wort „gemeinsam“ erwähnt. Das zeigt, was das Programm erst und vor allem speziell macht: Es ist das Gemeinsame - die doppelte Präsenz, der doppelte Spaß, den wir alle bei der Erfüllung des Programms gehabt haben und sicherlich auch in der Zukunft haben werden.

PhDr. Bc. Tomáš Svoboda, Ph.D.

Ústav translatologie, Filozofická fakulta, Univerzita Karlova tomas.svoboda@ff.cuni.cz 